FARIA, C.M.B.; COSTA, NLD.; SOARES, J.M.; PINTO, J.M.; LINS, J.M.; BRITO, L.T.L. Produção e qualidade de melão influenciados por matéria orgânica, nitrogênio e micronutrientes. Horticultura Brasileira, Brasília, v. 21, n. 1, p. 55-59, março 2003.

\title{
Produção e qualidade de melão influenciados por matéria orgânica, nitrogênio e micronutrientes ${ }^{1}$
}

\author{
Clementino Marcos Batista de Faria²; Nivaldo Duarte Costa² ${ }^{2}$ José Monteiro Soares $^{2}$; José Maria Pinto²; \\ José Maciel Lins; ${ }^{3}$ Luiza Teixeira de Lima Brito²
}

${ }^{2}$ Embrapa Semi-Árido, Caixa Postal 23, 56.300-970, Petrolina-PE, ${ }^{3}$ EBDA, Praça Imaculada Conceição, 28, 48.900-000, Juazeiro-BA. Email: clementi@cpatsa.embrapa.br

\section{RESUMO}

Avaliou-se a influência de nitrogênio, boro, molibdênio e zinco aplicados por fertirrigação e adição de esterco de gado ao solo sobre a produtividade e qualidade dos frutos de melão (Cucumis melo), em dois solos do Submédio São Francisco. Foram realizados seis experimentos nos anos de 1998, 1999 e 2000, sendo três num Latossolo Vermelho-amarelo, textura arenosa, em Petrolina (PE) e três num Vertissolo, em Juazeiro (BA). O delineamento experimental foi em blocos ao acaso, com quatro repetições e nove tratamentos, assim constituídos: 1- N1, 2- N2, 3- N3, 4- N1 + EG, 5- N2 + $\mathrm{EG}, 6-\mathrm{N} 2+(\mathrm{B}+\mathrm{Mo}+\mathrm{Zn}), 7-\mathrm{N} 2+(\mathrm{B}+\mathrm{Mo}), 8-\mathrm{N} 2+(\mathrm{B}+\mathrm{Zn}) \mathrm{e}$ 9- $\mathrm{N} 2+(\mathrm{Mo}+\mathrm{Zn})$, em que N1, N2 e N3 representam 0 ou 30, 80 e $160 \mathrm{~kg} \mathrm{ha}^{-1}$ de $\mathrm{N}$, respectivamente; EG, $20 \mathrm{~m}^{3} \mathrm{ha}^{-1}$ de esterco de gado; B, 1,1 kg ha ${ }^{-1}$ de B; Mo, 0,4 kg ha ${ }^{-1}$ de Mo e Zn, 4 kg/ha de Zn. $\mathrm{O} \mathrm{N}$ e os micronutrientes foram aplicados via água de irrigação. A produtividade do melão apresentou resposta a nitrogênio no Latossolo em 1998 e 2000 e no Vertissolo em 1999. Foi com 80 kg/ha de $\mathrm{N}$ que se obtiveram produtividade significativamente superior $(32,0 \mathrm{a}$ $40,2 \mathrm{t} \mathrm{ha}^{-1}$ ) em maior ocorrência dos experimentos. O EG provocou aumentos significativos apenas na produtividadede frutos, em 1998 e no peso médio dos frutos (PMF) em 2000, no Vertissolo. O teor de sólidos solúveis dos frutos aumentou com a aplicação de $\mathrm{N}$ no Latossolo, em 2000. Os micronutrientes não exerceram efeito significativo consistente. Em dois anos, o Vertissolo proporcionou PMF superior ao do Latossolo.

Palavras-chave: Cucumis melo, adubação, fertirrigação, produtividade, qualidade do fruto.

\section{ABSTRACT}

Effects of organic matter, nitrogen and micronutrients on yield and quality of melon

The effects of nitrogen, boron, molybdenum and zinc applied as fertigation, and cattle manure applied to the soil, were evaluated on the yield and quality of melon (Cucumis melo) in two types of soils of the Submedio São Francisco River Valley, Brazil. Six experiments were carried out in 1998, 1999 and 2000, three being in a red yellow Latosoil in Petrolina, and three in a Vertisoil, in Juazeiro, in a randomized complete block design with four replications and nine treatments, as follows: 1. N1; 2. N2; 3. N3; 4. N1 + C.M.; 5. N2+ C.M.; 6. N2 + B + Mo + Zn; 7. N2 + B + Mo; 8. N2 + B + Zn, and 9 . $\mathrm{N} 2+\mathrm{Mo}+\mathrm{Zn}$, where N1, N2 and N3 represent, respectively, 0 or 30,80 and $160 \mathrm{~kg} / \mathrm{ha}$ of $\mathrm{N}$; C.M. was $20 \mathrm{~m}^{3} \mathrm{ha}^{-1}$ of manure; $\mathrm{B}=1.1$ $\mathrm{kg} \mathrm{ha}^{-1} ; \mathrm{Mo}=0.4 \mathrm{~kg} \mathrm{ha}^{-1}$, and $\mathrm{Zn}=4 \mathrm{~kg} \mathrm{ha}^{-1}$. Nitrogen and the micronutrients were applied through irrigation water. Melon yield showed response to nitrogen in the Latosoil in 1998 and in 2000, and in the Vertisoil in 1999. Higher significant yields were obtained with $80 \mathrm{~kg} \mathrm{ha}^{-1}$ of $\mathrm{N}$, varying among experiments from 32.0 to 40.2 $\mathrm{t} \mathrm{ha}^{-1}$. Cattle manure caused significant increase only in yield in 1998 and in mean fruit weight in 2000 in the Vertisoil. Soluble solid contents in fruits increased with nitrogen application in the Latosoil in 2000. The micronutrients did not have significant effect on yield. In two years, mean fruit weight was higher in Vertisoil than in Latosoil.

Keywords: Cucumis melo; fertilization; fertigation; yield; fruit quality.

(Recebido para publicação em 23 de agosto de 2001 e aceito em 26 de novembro de 2002)

$\mathrm{O}$ melão é uma das espécies olerícolas de maior expressão econômica e social para a região Nordeste do Brasil. Atualmente, destacam-se como maiores produtores os estados do Rio Grande do Norte, Ceará, Pernambuco e Bahia, que contribuem com mais de $90 \%$ da produção nacional. Em Pernambuco e na Bahia a produção concentra-se no Vale do Submédio São Francisco.

O cultivo do melão exige adequada adubação para se obter produtividade alta com frutos de boa qualidade para atender às exigências dos mercados interno e externo. São raros os trabalhos sobre fertilização da cultura nessa região. Em dois experimentos realizados com o melão irrigado num Vertissolo, em Juazeiro, foi determinada a dose econômica de $74 \mathrm{~kg} \mathrm{ha}^{-1}$ de N, proporcionando incremento de $52 \%$ sobre a testemunha, mas não se verificou resposta à aplicação de $20 \mathrm{~m}^{3} \mathrm{ha}^{-1}$ de esterco de curral (Faria et al., 1994). Trabalhos de fertirrigação demonstraram que a dose adequada de $\mathrm{N}$ situa-se entre 80 e 139 $\mathrm{kg} \mathrm{ha}^{-1}$ (Pinto et al., 1995), que a uréia foi uma das fontes de $\mathrm{N}$ mais eficiente (Soares et al., 1999) e que a aplicação com frequência diária de $\mathrm{N}$, até o período de 42 dias após a germinação, foi um dos métodos mais adequados (Pinto $e t$ al., 1994).

Em outras regiões, Srinivas \& Prabhakar (1984) obtiveram aumento de $200 \%$ na produtividade de melão com a aplicação de $50 \mathrm{~kg} \mathrm{ha}^{-1}$ de N. Wilcox (1973) observou que as maiores produtividades de melão foram obtidas com as doses de 80 a $90 \mathrm{~kg} \mathrm{ha}^{-1}$ de N. Das várias doses de $\mathrm{N}$ testadas por Meisheri

${ }^{1}$ Convênio Embrapa/Petrobrás 
et al. (1984) na cultura do melão, $80 \mathrm{~kg}$ $\mathrm{ha}^{-1}$ foi considerada a melhor. Bhella \& Wilcox (1986) observaram que a aplicação de $67 \mathrm{~kg} \mathrm{ha}^{-1}$ de $\mathrm{N}$ ao solo mais 50 ou $100 \mathrm{mg} \mathrm{L}^{-1}$ de $\mathrm{N}$ na água de irrigação por gotejamento, proporcionou a produtividade mais alta do melão.

Além da produtividade, o nitrogênio também exerce efeito benéfico na qualidade dos frutos do melão, aumentando o peso e o teor de sólidos solúveis totais, conforme Srinivas \& Prabhakar (1984), Prabhakar et al. (1985) e Faria et al. (1994 e 2000).

A deficiência de alguns micronutrientes, como B, Mo e Zn, nos cultivos dos solos do Submédio São Francisco, tem sido questionada por agrônomos em observações de campo. Em oito experimentos de micronutrientes realizados nessa região, com algumas culturas irrigadas, Faria \& Pereira (1982) constataram deficiência de molibdênio no meloeiro cultivado num Vertissolo de Juazeiro, que tinha recebido sulfato de amônio em quantidade elevada. Sánchez (1997) relata que a carência de zinco em melão é pouco conhecida, mas descreve deficiências de boro e molibdênio.

Nos solos em que anteriormente as plantas não apresentavam resposta a micronutrientes aplicados, Zekri \& Koo (1992) verificaram que quando foram aplicados via fertirrigação, em baixa concentração, a cultura do citrus apresentou resposta positiva.

Esse trabalho teve o objetivo de avaliar a influência de nitrogênio, boro, molibdênio e zinco aplicados por fertirrigação e adição de esterco de gado ao solo sobre a produtividade e qualidade dos frutos de melão, em dois solos do Submédio São Francisco.

\section{MATERIAL E MÉTODOS}

O trabalho constou de seis experimentos de melão (Cucumis melo, L.) hibr. AF 682, realizados no Submédio São Francisco, no período entre outubro e dezembro dos anos de 1998, 1999 e 2000, em dois campos experimentais da Embrapa Semi-Árido, em Bebedouro (Petrolina), num Latossolo Vermelho amarelo, textura arenosa, e em Mandacaru (Juazeiro), num Vertissolo.
O Latossolo apresentava valores de: $\mathrm{pH}=6,3$ em $\mathrm{H}_{2} \mathrm{O} ; \mathrm{Ca}^{2+}=1,7 \mathrm{cmol} \mathrm{dm}^{-3}$; $\mathrm{Mg}^{2+}=0,6 \mathrm{c} \cdot \mathrm{mol}_{\mathrm{c}} \cdot \mathrm{dm}^{-3} ; \mathrm{Na}^{+}=0,06$ c. mol. $\mathrm{dm}^{-3} ; \mathrm{K}^{+}=0,30 \mathrm{c} . \mathrm{mol}_{\mathrm{c}} \cdot \mathrm{dm}^{-3} ; \mathrm{Al}^{3+}$ $=0,05 \mathrm{c} \cdot \mathrm{mol} \cdot \mathrm{dm}^{-3}$; C.E. $=0,37 \mathrm{dS} \cdot \mathrm{m}^{-1} \mathrm{e}$ $\mathrm{P}=42 \mathrm{mg} \mathrm{dm}^{-3}$ e o Vertissolo, valores de: $\mathrm{pH}=7,8$ em $\mathrm{H}_{2} \mathrm{O} ; \mathrm{Ca}^{2+},=27,0$ c.mol $\cdot \mathrm{dm}^{-3} ; \mathrm{Mg}^{2+}=7,0 \mathrm{c} \cdot \mathrm{mol} \cdot \mathrm{dm}^{-3} ; \mathrm{Na}^{+}$ $=0,19 \mathrm{c} \cdot \mathrm{mol} \cdot \mathrm{dm}^{-3} ; \mathrm{K}^{+}=0,34 \mathrm{c} \cdot \mathrm{mol} \cdot \mathrm{dm}^{-3}$; $\mathrm{Al}^{3+}=0,0$ c.mol $\cdot \mathrm{dm}^{-3}$; C.E. $=0,28 \mathrm{dS}$ $\mathrm{m}^{-1}$ e $\mathrm{P}=30 \mathrm{mg} \mathrm{dm}^{-3}$ segundo Embrapa (1997). Em 2000 analisaram-se amostras de solo para determinação também de micronutrientes segundo Raij et al. (1996), cujos valores foram: $\mathrm{B}=0,53 \mathrm{e}$ $0,23 \mathrm{mg} \mathrm{dm}^{-3} ; \mathrm{Cu}=2,1 \mathrm{e} 1,2 \mathrm{mg} \mathrm{dm}^{-3} ; \mathrm{Fe}$ $=17$ e $12 \mathrm{mg} \mathrm{dm}^{-3} ; \mathrm{Mn}=20$ e $15 \mathrm{mg} \mathrm{dm}^{-3}$ e $\mathrm{Zn}=33$ e $24 \mathrm{mg} \mathrm{dm}^{-3}$, para o Vertissolo e Latossolo, respectivamente.

O melão foi plantado no espaçamento de $1,8 \times 0,5 \mathrm{~m}$ e cultivado com irrigação pelo sistema de gotejamento em linha, com gotejadores espaçados de 1,0 m, vazão de $4 \mathrm{~L} \mathrm{~h}^{-1} \mathrm{e}$ pressão de $10 \mathrm{~m}$ de coluna de água. As irrigações foram feitas diariamente, com base na evaporação de água do tanque classe A e no coeficiente de cultura $(\mathrm{Kc})$.

$\mathrm{O}$ delineamento experimental foi em blocos ao acaso com quatro repetições e nove tratamentos: $1=\mathrm{N} 1,2=\mathrm{N} 2,3=$ $\mathrm{N} 3,4=\mathrm{N} 1+\mathrm{EG}, 5=\mathrm{N} 2+\mathrm{EG}, 6=\mathrm{N} 2$ $+\mathrm{B}+\mathrm{Mo}+\mathrm{Zn}, 7=\mathrm{N} 2+\mathrm{B}+\mathrm{Mo}, 8=$ $\mathrm{N} 2+\mathrm{B}+\mathrm{Zn}$ e $9=\mathrm{N} 2+\mathrm{Mo}+\mathrm{Zn}$, em que N1, N2 e N3 representam 0, 80 e $160 \mathrm{~kg} \mathrm{ha}^{-1}$ de $\mathrm{N}$, respectivamente; EG (esterco de gado $)=20 \mathrm{~m}^{3} \mathrm{ha}^{-1}$ de esterco de gado; $\mathrm{B}=1,1 \mathrm{~kg} \mathrm{ha}^{-1}$ de boro; Mo $=0,4 \mathrm{~kg} \mathrm{ha}^{-1}$ de molibdênio e $\mathrm{Zn}=4 \mathrm{~kg}$ ha $^{-1}$ de zinco. Em 1998 não houve os tratamentos 1 e 4 e em 1999 N1= $30 \mathrm{~kg}$ $\mathrm{ha}^{-1}$. A área útil da parcela foi de 25,2 $\mathrm{m}^{2}$. Todos os tratamentos receberam uma adubação uniforme com $80 \mathrm{~kg} \mathrm{ha}^{-1}$ de $\mathrm{P}_{2} \mathrm{O}_{5}$ e $80 \mathrm{~kg} \mathrm{ha}^{-1}$ de $\mathrm{K}_{2} \mathrm{O}$ nos dois experimentos. As fontes dos nutrientes foram o bórax em 1998 e 1999 e, ácido bórico em 2000; e uréia, superfosfato simples, cloreto de potássio, molibdato de sódio e sulfato de zinco para N, P, K, Mo e $\mathrm{Zn}$, respectivamente. O esterco de gado e o superfosfato simples foram aplicados em sulco antes do plantio. A uréia, o cloreto de potássio e as fontes dos micronutrientes foram parcelados em quantidades iguais para serem aplicados via água de irrigação. A frequiên- cia de aplicação foi a cada quatro dias para a uréia e cloreto de potássio e, semanal para os micronutrientes durante o período de três a 42 dias após a emergência (AE), três a 55 dias AE e entre a segunda e a sexta semana $\mathrm{AE}$, respectivamente.

No Latossolo foram necessárias duas colheitas para que o ciclo do meloeiro se completasse, enquanto no Vertissolo uma colheita foi suficiente, porque a cultura desenvolveu-se mais uniformemente, restando apenas poucos frutos não amadurecidos, não sendo necessária a segunda colheita. Foram avaliados a produtividade, o peso médio dos frutos (PMF) e o teor de sólidos solúveis totais (SST) do melão através da análise de variância e do teste Duncan segundo Snedecor \& Cochran (1971); também para os experimentos de 1999 foi feita a classificação dos frutos por tamanho ( $\mathrm{n}^{\circ}$ de frutos/caixa de embalagem, de dimensões de 48 x 35 x 15 cm).

\section{RESULTADOS E DISCUSSÃO}

Os dados de produtividade, PMF e SST dos frutos de melão, dos experimentos realizados no Latossolo encontram-se na Tabela 1 e dos experimentos no Vertissolo, na Tabela 2. A produtividade foi considerada alta em relação à média nacional (18.2 $\left.\mathrm{t} \mathrm{ha}^{-1}\right)$ e a do Nordeste brasileiro $\left(20,6 \mathrm{t} \mathrm{ha}^{-1}\right)$ segundo IBGE (2000). No ano de 2000 a produtividade foi mais alta do que nos outros anos em ambos solos, devido, principalmente à ocorrências de chuvas intensas nos últimos dez dias que antecederam a colheita $(173,8 \mathrm{~mm}$ no Vertissolo e $127,7 \mathrm{~mm}$ no Latossolo), fazendo com que a planta absorvesse mais água, tornando os frutos mais pesados. Os frutos com um teor de água mais elevado, apresentaram SST aparentemente mais baixo (Tabela 1). Esse fato foi mais pronunciado no Vertissolo (Tabela 2) porque, além da precipitação pluviométrica ter sido maior, a sua drenagem é menor. Com excessão do tratamento N1 no Latossolo, em 2000, os valores de SST foram considerados bons, uma vez que a exigência para o mercado é que os frutos tenham brix mínimo de 10 .

A produtividade do melão apresentou resposta a nitrogênio no Latossolo 
Tabela 1. Produtividade, peso médio do fruto (PMF) e sólidos solúveis totais (SST) do melão dos experimentos no Latossolo, nos três anos. Petrolina, Embrapa Semi Árido, 1998-2000.

\begin{tabular}{|c|c|c|c|c|c|c|c|c|c|}
\hline \multirow[b]{2}{*}{ Tratamento $^{2}$} & \multicolumn{3}{|c|}{1998} & \multicolumn{3}{|c|}{1999} & \multicolumn{3}{|c|}{2000} \\
\hline & $\begin{array}{c}\text { Produção } \\
\left(\text { kg ha }^{-1}\right)\end{array}$ & $\begin{array}{l}\text { PMF }^{1} \\
(\mathrm{~kg})\end{array}$ & $\begin{array}{l}\text { SST }^{1} \\
(\%)\end{array}$ & $\begin{array}{c}\text { Produção }^{1} \\
\left(\mathrm{~kg} \mathrm{ha}^{-1}\right)\end{array}$ & $\begin{array}{l}\text { PMF1 }^{1} \\
(\mathrm{~kg})\end{array}$ & $\begin{array}{c}\text { SST1 }^{1} \\
(\%)\end{array}$ & $\begin{array}{c}\text { Produção' } \\
\left(\text { kg ha }^{-1}\right)\end{array}$ & $\begin{array}{l}\text { PMF1 }^{1} \\
(\mathrm{~kg})\end{array}$ & $\begin{array}{r}\text { SST }^{1} \\
(\%)\end{array}$ \\
\hline$\overline{\mathrm{N} 1}$ & - & - & - & $31,5 b$ & $1,3 b$ & $12,2 a b$ & $23,9 c$ & $1,7 a$ & $9,7 b$ \\
\hline N2 & $32,4 a$ & $1,7 a$ & $11,5 a$ & $36,3 a b$ & $1,5 a$ & $11,9 a b$ & $40,2 a b$ & $1,7 a$ & $10,0 a b$ \\
\hline N3 & $22,9 b$ & $1,4 a$ & $11,6 a$ & $36,7 a b$ & $1,5 a$ & $11,7 a b c$ & $40,2 a b$ & $1,8 a$ & $11,1 \mathrm{a}$ \\
\hline $\mathrm{N} 1+\mathrm{MO}$ & - & - & - & $32,2 b$ & $1,3 b$ & $11,4 a b c$ & - & - & - \\
\hline $\mathrm{N} 2+\mathrm{MO}$ & $30,8 a$ & $1,6 a$ & $12,2 a$ & $32,6 b$ & $1,5 a$ & $10,7 \quad c$ & $44,7 a$ & $1,8 a$ & $10,6 a b$ \\
\hline $\mathrm{N} 2+(\mathrm{B}+\mathrm{Mo}+\mathrm{Zn})$ & $28,6 a b$ & $1,6 a$ & $11,6 a$ & $36,3 a b$ & $1,4 a$ & $11,8 a b$ & $36,6 b$ & $1,7 a$ & $10,8 a b$ \\
\hline $\mathrm{N} 2+(\mathrm{B}+\mathrm{Mo})$ & $37,7 a$ & $1,5 a$ & $11,6 a$ & $35,3 a b$ & $1,4 a$ & $12,3 a$ & $35,2 b$ & $1,8 a$ & $11,1 \mathrm{ab}$ \\
\hline $\mathrm{N} 2+(\mathrm{B}+\mathrm{Zn})$ & $32,8 a$ & $1,6 a$ & $12,2 a$ & $33,6 a b$ & $1,5 a$ & $11,1 \mathrm{bc}$ & $40,1 a b$ & $1,8 a$ & $11,3 a$ \\
\hline $\mathrm{N} 2+(\mathrm{Mo}+\mathrm{Zn})$ & $31,8 a$ & $1,6 a$ & $11,6 a$ & $38,8 a$ & $1,4 a b$ & $12,1 \mathrm{ab}$ & $36,1 b$ & $1,6 a$ & $10,9 a b$ \\
\hline C.V.\% & 13,6 & 11,9 & 5,4 & 10,2 & 5,7 & 5,7 & 12,5 & 6,8 & 7,7 \\
\hline
\end{tabular}

${ }^{1}$ Valores seguidos da mesma letra, na mesma coluna, não diferem pelo teste Duncan a 5\% de probabilidade.

${ }^{2} \mathrm{~N} 1, \mathrm{~N} 2$ e N3=0, 80 e $160 \mathrm{~kg} / \mathrm{ha}$ de N, respectivamente; em $1999 \mathrm{~N} 1=30 \mathrm{~kg} \mathrm{ha}^{-1}$; MO=20 $\mathrm{m}^{3} \mathrm{ha}^{-1}$ de esterco de gado; B=1,1 kg ha-1 de B; $\mathrm{Mo}=0,4 \mathrm{~kg} \mathrm{ha}^{-1}$ de Mo; $\mathrm{Zn}=4 \mathrm{~kg} \mathrm{ha}^{-1}$ de $\mathrm{Zn}$.

Tabela 2. Produtividade, peso médio do fruto (PMF) e sólidos solúveis totais (SST) do melão dos experimentos no Vertissolo, nos três anos. Petrolina, Embrapa Semi Árido, 1998-2000.

\begin{tabular}{|c|c|c|c|c|c|c|c|c|}
\hline \multirow[b]{2}{*}{ Tratamento $^{2}$} & \multicolumn{3}{|c|}{1998} & \multicolumn{2}{|l|}{1999} & \multicolumn{3}{|c|}{2000} \\
\hline & $\begin{array}{c}\text { Produção } \\
\left(\mathrm{kg} \mathrm{ha}^{-1}\right)\end{array}$ & $\begin{array}{l}\text { PMF1 }^{1} \\
(\mathrm{~kg})\end{array}$ & $\begin{array}{l}\text { SST }^{1} \\
(\%)\end{array}$ & $\begin{array}{c}\text { Produção }^{1} \\
\left(\mathrm{~kg} \mathrm{ha}^{-1}\right)\end{array}$ & $\begin{array}{l}\mathrm{PMF}^{1} \\
(\mathrm{~kg})\end{array}$ & $\begin{array}{c}\text { Produção }{ }^{1} \\
\left(\text { kg ha }^{-1}\right)\end{array}$ & $\begin{array}{c}\mathrm{PMF}^{1} \\
(\mathrm{~kg})\end{array}$ & $\begin{array}{r}\text { SST } \\
(\%)\end{array}$ \\
\hline N1 & - & - & - & $22,0 d$ & $1,4 \mathrm{de}$ & $35,7 a$ & $1,8 b$ & $11,2 a$ \\
\hline N2 & $28,8 b$ & $1,6 b$ & $11,8 a$ & $32,0 a$ & $1,8 a$ & $35,7 a$ & $1,6 b$ & $10,4 a$ \\
\hline N3 & $32,5 a b$ & $1,7 a b$ & $11,5 a$ & $28,4 a b c$ & $1,7 a b$ & $38,9 a$ & $1,7 b$ & $11,1 \mathrm{a}$ \\
\hline $\mathrm{N} 1+\mathrm{MO}$ & - & - & - & $24,4 \mathrm{~cd}$ & $1,3 e$ & - & - & - \\
\hline $\mathrm{N} 2+\mathrm{MO}$ & $37,7 a$ & $1,8 a b$ & $12,6 a$ & $29,4 a b$ & $1,6 \mathrm{bcd}$ & $37,9 a$ & $2,1 \mathrm{a}$ & $10,6 a$ \\
\hline $\mathrm{N} 2+(\mathrm{B}+\mathrm{Mo}+\mathrm{Zn})$ & $30,6 b$ & $1,8 a$ & $12,5 a$ & $25,0 \mathrm{bcd}$ & $1,4 \mathrm{cde}$ & $34,5 a$ & $1,6 b$ & $10,1 a$ \\
\hline $\mathrm{N} 2+(\mathrm{B}+\mathrm{Mo})$ & $33,4 a b$ & $1,9 a$ & $11,7 a$ & $29,3 a b$ & $1,5 \mathrm{cde}$ & $38,9 a$ & $1,7 b$ & $10,6 a$ \\
\hline $\mathrm{N} 2+(\mathrm{B}+\mathrm{Zn})$ & $29,0 b$ & $1,7 a b$ & $11,5 a$ & $27,8 a b c$ & $1,6 a b c$ & $33,9 a$ & $1,8 b$ & $10,8 a$ \\
\hline $\mathrm{N} 2+(\mathrm{Mo}+\mathrm{Zn})$ & $33,7 a b$ & $1,7 a b$ & $12,1 \mathrm{a}$ & $26,1 \mathrm{bcd}$ & $1,6 \mathrm{bcd}$ & $34,9 a$ & $1,6 b$ & $11,0 a$ \\
\hline C.V.\% & 10,3 & 7,4 & 11,9 & 10,9 & 8,3 & 9,7 & 10,0 & 7,8 \\
\hline
\end{tabular}

${ }^{1}$ Valores seguidos da mesma letra, na mesma coluna, não diferem pelo teste Duncan a 5\% de probabilidade.

${ }^{2} \mathrm{~N} 1$, N2 e N3=0, 80 e $160 \mathrm{~kg} \mathrm{ha}^{-1}$ de N, respectivamente; em $1999 \mathrm{N1}=30 \mathrm{~kg} \mathrm{ha}^{-1} ; \mathrm{MO}=20 \mathrm{~m}^{3} \mathrm{ha}^{-1}$ de esterco de gado; $\mathrm{B}=1,1 \mathrm{~kg}$ ha ${ }^{-1} \mathrm{de} \mathrm{B}$; $\mathrm{Mo}=0,4 \mathrm{~kg} \mathrm{ha}^{-1}$ de $\mathrm{Mo} ; \mathrm{Zn}=4 \mathrm{~kg} \mathrm{ha}^{-1}$ de $\mathrm{Zn}$.

em dois anos (Tabela 1) e no Vertissolo, em 1999 (Tabela 2). O Latossolo por ser de textura arenosa, apresenta probabilidade mais elevada de deficiência de $\mathrm{N}$ que o Vertissolo de textura argilosa. No Latossolo em 1998, a produtividade decresceu significativamente com o aumento da dose de $\mathrm{N}$ de 80 para $160 \mathrm{~kg}$ $\mathrm{ha}^{-1}$, atribuindo-se a desequilíbrio nutricional na planta provocado pela dose mais elevada. Em 2000, $80 \mathrm{~kg} \mathrm{ha}^{-1}$ de $\mathrm{N}$ condicionaram um rendimento de frutos maior que o da testemunha (sem $\mathrm{N})$ e semelhante ao obtido com $160 \mathrm{~kg}$ ha $^{-1}$ de N. No Vertissolo em 1999, $80 \mathrm{~kg}$ $\mathrm{ha}^{-1}$ proporcionou uma produtividade significativamente superior a de $30 \mathrm{~kg}$ ha $^{-1}$ de N. Os efeitos das doses de nitrogênio foram semelhantes aos resultados obtidos por Faria et al. (1994); Pinto et al (1995); Faria et al. (2000) na mesma região e por Wilcox (1973), Meisheri et al. (1984) em outras regiões.

No Vertissolo, em 1999, o PMF com 80 e $180 \mathrm{~kg} \mathrm{ha}^{-1}$ de $\mathrm{N}$ alcançou valores significativamente mais elevados do que com $30 \mathrm{~kg} \mathrm{ha}^{-1}$. No Latossolo em 2000 , o SST com $160 \mathrm{~kg} \mathrm{ha}^{-1}$ foi superior ao do nível zero de N. Essas influências na qualidade do fruto exercidas pelo nitrogênio foram observadas em outros trabalhos (Srinivas \& Prabhakar, 1984; Prabhakar et al., 1985; Faria et al., 1994; 2000).

O esterco de gado provocou aumentos significativos somente no Vertissolo (Tabela 2), na produtividade em $1998 \mathrm{e}$ no PMF em 2000. Em alguns casos houve até efeitos negativos do esterco, como o PMF no Vertissolo, em 1999 e no SST, no Latossolo, em 1999. Isso não era esperado acontecer em solos de região semi-árida, pobres em matéria orgânica. No entanto, considerando que a cultura do melão tem um ciclo muito curto (70 dias), talvez não houve tempo sufi- 
Tabela 3. Classificação do tamanho dos frutos de melão no Latossolo. Petrolina, Embrapa Semi Árido, 1998-2000.

\begin{tabular}{lccccc}
\hline \multirow{2}{*}{ Tratamento $^{1}$} & \multicolumn{5}{c}{ Tipo (no de frutos/caixa) $^{\mathbf{2}}$} \\
\cline { 2 - 6 } & $\mathbf{6}$ & $\mathbf{7}$ & $\mathbf{8}$ & $\mathbf{9}$ & $\mathbf{1 0}$ \\
\hline $\mathrm{N} 1$ & 3 & 75 & 54 & 67 & 51 \\
$\mathrm{~N} 2$ & 17 & 78 & 79 & 52 & 24 \\
$\mathrm{~N} 3$ & 17 & 67 & 70 & 62 & 31 \\
$\mathrm{~N} 1+\mathrm{MO}$ & 7 & 64 & 56 & 60 & 61 \\
$\mathrm{~N} 2+\mathrm{MO}$ & 6 & 71 & 60 & 50 & 24 \\
$\mathrm{~N} 2+(\mathrm{B}+\mathrm{MO}+\mathrm{Zn})$ & 17 & 78 & 64 & 62 & 33 \\
$\mathrm{~N} 2+(\mathrm{B}+\mathrm{MO})$ & 10 & 83 & 62 & 69 & 23 \\
$\mathrm{~N} 2+(\mathrm{B}+\mathrm{Zn})$ & 11 & 64 & 62 & 61 & 30 \\
$\mathrm{~N} 2+(\mathrm{MO}+\mathrm{Zn})$ & 14 & 59 & 76 & 94 & 36 \\
\hline TOTAL & 107 & 639 & 583 & 577 & 316 \\
\hline
\end{tabular}

${ }^{1} \mathrm{~N} 1, \mathrm{~N} 2$ e N3=0, 80 e $160 \mathrm{~kg} \mathrm{ha}^{-1}$ de N, respectivamente; em $1999 \mathrm{N1}=30 \mathrm{~kg} \mathrm{ha}^{-1} ; \mathrm{MO}=20 \mathrm{~m}^{3} \mathrm{ha}^{-1}$ de esterco de gado; $\mathrm{B}=1,1 \mathrm{~kg}$ ha ${ }^{-1}$ de $\mathrm{B}$; $\mathrm{Mo}=0,4 \mathrm{~kg} \mathrm{ha}^{-1} \mathrm{de} \mathrm{Mo} ; \mathrm{Zn}=4 \mathrm{~kg} \mathrm{ha}^{-1} \mathrm{de} \mathrm{Zn}$.

${ }^{2}$ Caixa de embalagem com as dimensões de $48 \times 35$ x $15 \mathrm{~cm}$

Tabela 4. Classificação do tamanho dos frutos de melão no Vertissolo. Petrolina, Embrapa Semi Árido, 1998-2000.

\begin{tabular}{lccccc}
\hline \multirow{2}{*}{ Tratamento $^{1}$} & \multicolumn{5}{c}{ Tipo (no de frutos/caixa) $^{\mathbf{2}}$} \\
\cline { 2 - 6 } & $\mathbf{6}$ & $\mathbf{7}$ & $\mathbf{8}$ & $\mathbf{9}$ & $\mathbf{1 0}$ \\
\hline $\mathrm{N} 1$ & 12 & 31 & 43 & 31 & 40 \\
$\mathrm{~N} 2$ & 41 & 64 & 37 & 9 & 30 \\
$\mathrm{~N} 3$ & 43 & 55 & 34 & 14 & 0 \\
$\mathrm{~N} 1+\mathrm{MO}$ & 23 & 34 & 34 & 42 & 48 \\
$\mathrm{~N} 2+\mathrm{MO}$ & 32 & 43 & 54 & 15 & 40 \\
$\mathrm{~N} 2+(\mathrm{B}+\mathrm{MO}+\mathrm{Zn})$ & 25 & 44 & 36 & 29 & 42 \\
$\mathrm{~N} 2+(\mathrm{B}+\mathrm{MO})$ & 31 & 52 & 49 & 30 & 25 \\
$\mathrm{~N} 2+(\mathrm{B}+\mathrm{Zn})$ & 40 & 47 & 43 & 17 & 30 \\
$\mathrm{~N} 2+(\mathrm{MO}+\mathrm{Zn})$ & 26 & 51 & 37 & 20 & 34 \\
\hline TOTAL & 273 & 421 & 367 & 207 & 289 \\
\hline
\end{tabular}

${ }^{1} \mathrm{~N} 1, \mathrm{~N} 2$ e N3=0, 80 e $160 \mathrm{~kg} \mathrm{ha}^{-1}$ de N, respectivamente; em $1999 \mathrm{~N} 1=30 \mathrm{~kg} \mathrm{ha}^{-1} ; \mathrm{MO}=20 \mathrm{~m}^{3} \mathrm{ha}^{-1}$ de esterco de gado; $\mathrm{B}=1,1 \mathrm{~kg}$ ha ${ }^{-1}$ de $\mathrm{B}$; $\mathrm{Mo}=0,4 \mathrm{~kg} \mathrm{ha}^{-1}$ de Mo; $\mathrm{Zn}=4 \mathrm{~kg} \mathrm{ha}^{-1}$ de $\mathrm{Zn}$.

${ }^{2}$ Caixa de embalagem com as dimensões de 48 x 35 x $15 \mathrm{~cm}$

ciente para se beneficiar dos efeitos do esterco de gado, que dependem do seu grau de decomposição no momento do plantio e no início do desenvolvimento das plantas. Em relação à cebola, que tem ciclo vegetativo maior (120 dias), Faria et al. (1981) obtiveram respostas significativas à aplicação de esterco de gado em dois solos da região, durante três anos, com incrementos de 20 a $133 \%$ na produtividade de bulbos.

Os micronutrientes não exerceram nenhum efeito significativo nas características avaliadas, a não ser sobre o SST no Latossolo, em 1999, em que o tratamento com a omissão de molibdênio proporcionou valor inferior ao do tratamento com boro + molibdênio mas, por outro lado, essa diferença não foi constatada em relação à aplicação completa dos três micronutrientes. Em outras situações da região, onde houve aplicações de sulfato de amônio em excesso, em solos com drenagem deficiente, o melão respondeu positivamente a molibdênio (Faria \& Pereira, 1982). A baixa ocorrência de resposta das plantas a micronutrientes foi constatada por Faria \& Pereira (2000) em aplicações no solo. Através da aplicação via água de irrigação, esperava-se que houvesse resposta da planta aos micronutrientes aplicados, conforme obtiveram Zekri \& Koo (1992), apesar dos teores de micronutrientes dos solos dos experimentos serem considerados médio a alto, conforme Raij et al. (1996).

Em análise conjunta para os dois solos, constatou-se que o PMF de 1,7 $\mathrm{kg}$ em 1998 e de 1,6 kg em 1999 obtidos no Vertissolo, foram significativamente superiores aos obtidos no Latossolo, 1,6 kg em 1998 e 1,4 kg em 1999, o que é atribuído, em parte, aos maiores teores de bases trocáveis $(\mathrm{Ca}$, $\mathrm{Mg}, \mathrm{K}$ ) do primeiro solo.

As Tabelas 3 e 4 contêm a classificação dos frutos por tamanho dos expe- 
rimentos em 1999, no Latossolo e no Vertissolo, respectivamente. A maioria dos frutos, principalmente no Latossolo, foram dos tipos 7 e 8 , que são os mais aceitos pelo mercado interno. No Vertissolo também houve ocorrência de frutos muito grandes, tipo $6(17,5 \%)$, que são aceitos em alguns mercados internos locais e de frutos pequenos, tipo 10 $(18,5 \%)$, aceitos pelo mercado externo. O tipo 9, que também é aceito pelo mercado externo, surgiu com uma frequência alta $(25,9 \%)$ no Latossolo. Em relação aos tratamentos, a única distinção consistente que se observa é para o N1, onde ocorreu a menor percentagem de frutos do tipo 6 (1,2 e 7,6\%) e elevada percentagem de frutos tipo 10 (20,4 e 25,5\%) para o Latossolo e Vertissolo, respectivamente. Isso está relacionado com a influência positiva do nitrogênio no PMF (Faria et al. 1994; 2000).

Conclui-se que a probabilidade de se obter ganhos significativos na produtividade e qualidade do melão no Submédio São Francisco com a adição de esterco de gado é pequena, o mesmo acontecendo com a adição de boro, molibdênio e zinco. A dose de $80 \mathrm{~kg} \mathrm{ha}$ ${ }^{1}$ de $\mathrm{N}$ é suficiente para se obter produtividade em torno de $35 \mathrm{t} \mathrm{ha}^{-1}$ de frutos com boa qualidade. No Vertissolo obtém-se frutos iguais ou mais pesados que os do Latosolo.

\section{LITERATURA CITADA}

BHELLA, H.S.; WILCOX, G.E. Yield composition of muksmelon as influenced by preplant and trickle applied nitrogen. Hortscience, v. 21, n. 1 . p. $86-88,1986$

EMBRAPA. Centro Nacional de Pesquisa de Solos (Rio de Janeiro,RJ). Manual de métodos de análises de solo. 2. ed. rev. atual. Rio de Janeiro, 1997. 212 p. (EMBRAPA-CNPS. Documentos; $1)$.

FARIA, C.M.B.; COSTA, N.D.; PINTO, J.M.; BRITO, L.T.L.; SOARES, J.M. Níveis de nitrogênio por fertirrigação e densidade de plantio na cultura do melão em um Vertissolo. Pesquisa Agropecuária Brasileira, Brasília, v. 35, n. 3, p. 491-495, 2000

FARIA, C.M.B.; MENEZES, D.; CANDEIA, J.A Influência da adubação orgânica e mineral nitrogenada no rendimento da cebola em dois solos do Submédio São Francisco. Pesquisa Agropecuária Pernambucana, Recife, v. 5, p. 7179, 1981.

FARIA, C.M.B.; PEREIRA, J.R. Ocorrência do "amarelão" no meloeiro e seu controle. PetrolinaPE, EMBRAPA-CPATSA, 1982. 2 p. (EMBRAPA-CPATSA, Comunicado Técnico, 8) FARIA, C.M.B.; PEREIRA, J.R.; POSSÍDIO, E.L. Adubação orgânica e mineral na cultura do melão em um Vertissolo do Submédio São Francisco. Pesquisa Agropecuária Brasileira, Brasília, v. 29, n. 2, p. 191-197,1994

FARIA, C.M.B.; PEREIRA, J.R. Resposta de cultivares irrigadas à aplicação de micronutrientes no Submédio São Francisco. Pesquisa Agropecuária Brasileira, Brasília, v. 35, n. 6, p. 1275-1280, 2000. IBGE. Produção agrícola. Disponível: site Sistema IBGE de recuperação automática - SIDRA (2000). URL: http://sidra.ibge.gov.br/cgi-bim/v/m/ pamltap. consultado em 12 de setembro 2000.
MEISHERI, T.G. ; JADAV, K.V. ; PATEL, J.J. ; PATEL, D.P. Effect of different levels of nitrogen and phosphorus on the fruit yield to muskmelon (Cucumis melo) var. GMM - 1 GAU Research Journal, v. 9 n. 2, p. 10-13, 1984.

PINTO, J.M.; SOARES, J.M.; PEREIRA, J.R.; CHOUDHURY, E.N.; CHOUDHURY, M.M. Efeitos de períodos e de frequências da fertirrigação nitrogenada na produção do melão. Pesquisa Agropecuária Brasileira, Brasília v. 29, n. 9, p. 1345-1350, 1994.

PINTO, J.M.; SOARES, J.M.; COSTA, N.D.; BRITO, L.T.L.; PEREIRA, J.R. Aplicação de N e $\mathrm{K}$ via água de irrigação em melão. Horticultura Brasileira, Brasília, v. 13, n. 2, p. 192-195, 1995.

PRABHAKAR, B.S.; SRINIVAS, K.; SHUKLA,

V. Yield and quality of muskmelon (cv. Hara madhu) in relation to spacing and fertilization. Progressive Horticulture, v. 17, n. 1, p. 51-55, 1985. RAIJ, B.; CANTARELLA, H.; QUAGGIO, J.A.; FURLANI, A.M.C. Recomendações de adubação e calagem para o Estado de São Paulo, 2. ed. Campinas, Instituto Agronômico \& Fundação IAC, 1996. 285 p. (Boletim Técnico 100).

SÁNCHEZ, L.R. Fertilización del melón en riego por goteo. In: NAMESNY, A., Coord. Melones. Reus, Ediciones de Jorticultura, S.L., 1997. p. 85-93.

SOARES, J.M.; BRITO, L.T.L.; COSTA, N.D.; MACIEL, J.L. FARIA, C.M.B. Efeito de fertilizantes nitrogenados na produtividade de melão. Pesquisa Agropecuária Brasileira, Brasília, v. 34, n. 7, p. 1139-1143, 1999.

SNEDECOR, G.W.; COCHRAN, W.G. Métodos estadísticos. México: Continental, 1971. 703 p. SRINIVAS, K.; PRABHAKAR, B.S. Response of muskmelon (Cucumis melo L.) to varying levels of spacing and fertilizers. Singapore Journal of Primary Industries, v. 12, n. 1, p. 56-61, 1984.

WILCOX, G.E. Muskmelon response to rates and sources of nitrogen. Agronomy Journal, v. 65, n. 5, p. 694-697, 1973

ZEKRI, M.; KOO, R.C.J. Application of micronutrients to citrus trees through microirrigation systems. Journal of Plant Nutrition, v. 15, n. 11, p. 2517-2529, 1992. 